\title{
A Novel Machine Learning Model for the Detection of Epilepsy and Epileptic Seizures Using Electroencephalographic Signals Based on Chaos and Fractal Theories
}

\author{
Zayneb Brari iD and Safya Belghith (iD \\ RISC Laboratory, École Nationale d’Ingénieurs de Tunis, Université de Tunis El Manar, BP 37, 1002, Le Bélvédère, \\ Tunis 1002, Tunisia \\ Correspondence should be addressed to Zayneb Brari; brarizayneb@gmail.com
}

Received 30 April 2021; Revised 2 July 2021; Accepted 27 July 2021; Published 9 August 2021

Academic Editor: Dilbag Singh

Copyright (C) 2021 Zayneb Brari and Safya Belghith. This is an open access article distributed under the Creative Commons Attribution License, which permits unrestricted use, distribution, and reproduction in any medium, provided the original work is properly cited.

\begin{abstract}
Machine learning is an expanding research area. Its main application is in the medical field and particularly the detection of epilepsy and epileptic seizures through electroencephalographic signals (EEG). It aims to design an intelligent framework that enables an immediate diagnosis of this disease without neurological consultation and thus saves the lives of the epileptic patients by detecting seizures and warning them before it happens. However, as a real-time application, this kind of framework faces several challenges such as accuracy, fast responses, and optimal memory usage. Within this context, our work was carried out. We propose a new machine learning framework based on chaos and fractal theories. Two main novelties are presented in this paper. Firstly, we propose a new method for signal preprocessing, and we reconstruct new versions of studied EEG signals using derivative determination and chaotic injection. Secondly, we suggest a new method for fractal analysis using Higuchi fractal dimension (HFD). In fact, HFDs extracted from EEG derivatives lead to detect epilepsy, whereas HFDs extracted from EEG with a chaotic signal injection lead to seizure detection. In addition, feature fusion helped to linearize all classification problems. An experimental study using the Bonn EEG database proves the efficiency of our contributions in comparison to published research. An accuracy of $100 \%$ was achieved in different classification cases using few features and a simple linear classifier.
\end{abstract}

\section{Introduction}

Epilepsy is a dangerous brain disorder that can put patients' lives at high risks. EEG is the basic examination for epilepsy therapy since it leads to significant results, is easy to achieve, and is not expensive. However, the exploitation of EEG traces is time consuming and requires neurologists for the interpretation. Over the past decades, technology evolution has led to the possibility of recording EEG in the digital data form for computer analysis. After that, developing automatic systems for epilepsy detection and epileptic patient monitoring became an interesting research field, with a lot of challenges for a real-time application. More than the robustness and efficiency, developed systems seek to overcome some key points. Basically, learning and predicting times must be reduced which is important in case we need to update our model and to have a fast prediction of the treated state in order to take the necessary preoccupations. Otherwise, memory requirements impose data reduction and an optimal choice of the data processing method. Nevertheless, EEGs are complex signals due to their irregularity, nonlinearity, and nonstationarity and are obviously disturbed by noises. For all these reasons, the development of machine learning models based on the EEG is a hard task. According to a literature review, EEG processing goes through the following steps. The first one is signal preprocessing for noise removal using different filtering methods, while the second step is about feature extraction and selection to reduce the data vector using the time domain or a transformed or decomposed version of the studied signal in other domains. The final step is the classification which is used to detect the patient brain state. Over the last century, many published 
research studies proved that the complexity of EEG signals is not from a random aspect, it is rather chaotic with a fractal structure [1-3]. Then, chaos and fractal theories have been widely used to study EEG signals. In [4], authors extracted features using signal transformation in the frequency domain, fast Fourier transform (FFT), and then they calculated relative intensity ratio (RIR) for different frequencies. Fractal dimension is also calculated with Petrosian (FDP) and HFD methods. Moreover, Hjorth parameters (HPs) are used to evaluate signal complexity. Sharma et al. [5] decomposed the EEG signal using the analytic time-frequency flexible wavelet transform (ATFFWT) in 17 subbands and extracted HFD for each one. Then, these features were used in the support vector machine (SVM) classifier. In [6], Sharma and Pachori decomposed the signal with the tunable Q-factor wavelet transform (TQFWT), extracted HFD for different subbands, and evaluated them using the least-squares support vector machine (LS-SVM). Sharma et al. proposed a novel approach [7] in 2019 using the biorthogonal wavelet transform (BOWT), used HFD with other nonlinear features (NLfeats) such as Shannon, Renyi, and Fuzzy entropies (ShanEn, ReEn, FuzEn) and energy (En), and classified signals with the SVM. Fractal and nonlinear analyses were used in Zhang and Chen's work [8], who calculated HFD, ReEn, and Hurst exponent (HE) from the EEG and extracted six temporal statistic features (TSFs) from the product function of local mean decomposition (LMD); these features were classified through the SVM. The same classifier is used in [9] with multifractal analysis (MFA). Recently, Jiang et al. [10] decomposed the signal through the scattering transform (ScT), and then they calculated the entropy by two methods, FuzEn and log energy entropy (LogEEn). Later, SVM was used for the classification.

According to this literature research, we have found that nonlinear analysis and fractal dimension are interesting tools for characterizing EEG signals. However, in all cited papers, [4-10], different signal decomposition methods were used that lead to obtaining a high-dimensional feature vector, which is disadvantageous in real applications, time consumption, and memory usage. In our previous work [11], we have proved the usefulness of derivatives to extract information from EEG. We extracted the logarithm of variance (LogVar) from EEG signal as well as its first two derivatives. Then, we used the kernel trick (KT) to linearize features' space. Achieved accuracy in this paper is important, whereas our work is limited to solving a part of all the stateof-the-art classification problems using the same database.

In this work, we propose two new methods for machine learning model building based on fractal and chaos theories and use EEG signal derivatives. Features are extracted from a transformed version of EEG signals. In fact, a chaotic signal injection allows seizure detection. In addition to this, fractal study applied to EEG and its derivatives and a chaotic injection to the extracted derivatives permit to qualify perfectly healthy subjects as well as epilepsy detection. Finally, a feature fusion, using a nonlinear function, is applied to extracted features from all signal versions to solve all the classification cases studied in our work. Our contributions for EEG signal processing through this paper are interesting in terms of the achieved accuracy, simplicity, and run time compared to the other published works, which use the same database studied in this paper.

This paper is organized as follows: we will start by presenting the used database and several works prepared for the same objective as our work in Section 2. Then, we will introduce our proposed method and the achieved results and discuss our work in Section 3. Finally, a summary of the paper with a conclusion and perspectives will be given in Section 5 .

\section{Materials and Methods}

2.1. EEG Database. In our study, we use an open-source database published by Bonn University and described in [12]; it contains 5 subsets ( $, \mathrm{O}, \mathrm{N}, \mathrm{F}$, and S), and each subset contains 100 signals with 4097 samples. $Z$ and $O$ are extracted from healthy $(\mathrm{H})$ subjects with, respectively, open and closed eyes. $\mathrm{N}$ and $\mathrm{F}$ are recorded from epileptic (E) subjects from different brain zones. $\mathrm{S}$ is saved during epileptic seizures (ES). This database was used in different published works. In Table 1, we present a selection of performed experiments (Exps) with the used subset (U-Sub) that we will study later.

2.2. Proposed Machine Learning Model. The method proposed in this manuscript to detect a patient's brain state is illustrated in Figure 1; we went through five steps:

(i) Signal preprocessing: we reconstruct three new versions of the processed signal. Then, we introduce new time series, defined as follows:

$$
\begin{aligned}
& x_{1}=e+\alpha \cdot c, \\
& x_{2}=d^{3} e+\alpha \cdot c, \\
& x_{3}=d^{3} e,
\end{aligned}
$$

where $e$ is the studied EEG signal, $c$ is the chaotic signal extracted from the logistic map and $\alpha$ is its amplitude, and $d^{3}$ is the third derivative of the $e$ signal.

(ii) Feature extraction: we extract the Higuchi fractal dimension from the EEG signal, $e$, and the tree reconstructed versions, $x_{1}, x_{2}$, and $x_{3}$.

(iii) Feature space reduction: we removed irrelevant features and selected the most significant ones that solve classification problems. Mainly, feature reduction is an important step to reduce the used memory that is beneficial to save the trained version. In our work, it was also beneficial for the presentation of features in $2 \mathrm{D}$ and $3 \mathrm{D}$ spaces that will be helpful in the following steps.

(iv) Feature space linearization: extracted features from the four versions of the studied signal are used to reconstruct a new feature space based on the feature fusion approach. Since our goal is to classify three types of EEG signals ( $\mathrm{H}, \mathrm{E}$, and $\mathrm{ES}$ ), we propose to 
TABLE 1: EEG classification problems.

\begin{tabular}{lcc}
\hline Experiments & Description & U-Sub \\
\hline Exp 1 & H vs. E & $(\mathrm{Z}$ and/or O) vs. (N and/or F) \\
Exp 2 & H vs. ES & $(\mathrm{Z}$ and/or O) vs. S \\
Exp 3 & E vs. ES & $(\mathrm{C}$ and/or O) vs. S \\
Exp 4 & (H and E) vs. ES & $((\mathrm{Z}$ and/or O), (N and/or F)) vs. S \\
Exp 5 & H vs. (E and ES) & $(\mathrm{Z}$ and/or O) vs. ( $\mathrm{N}$ and/or F), S) \\
Exp 6 & H vs. E vs. ES & $(\mathrm{Z}$ and/or O) vs. (N and/or F) vs. S \\
\hline
\end{tabular}

divide classification problems studied in our paper into three categories:

(i) One versus one $(\operatorname{Exp} 1, \operatorname{Exp} 2$, and $\operatorname{Exp} 3)$ : to solve the binary classification problem using two types of signals. Selected features from the previous step were applied to design the linear feature space using "feature fusion 1."

(ii) One versus all (Exp 4 and Exp 5): to solve the binary classification problem using three types of signals. We use a transform version of linear features selected from the reconstructed space in (i) to reduce the feature space in "feature fusion 2."

(iii) Multiclass (Exp 6): to solve the multiclass classification problem using three types of signals. The output of the previous step yields to simplify this problem based on "feature fusion 3."

(v) Classification: through the previous steps, we managed to solve all the classification problems deliberated in our work with a linear classifier.
2.3. Fractal Dimension. EEG signal processing through FD is widely used to detect many neurological disorders such as Alzheimer's [13], mental disorders [4], and epilepsy [4-11]. FD is calculated through different methods, Katz algorithm, multiresolution box-counting, Higuchi method [14], etc. The most used method in EEG signal processing is HFD [15]. HFD was firstly defined in his paper [14]; its determination follows the following steps:

(i) From time series $Y_{N}=Y(1), Y(2), \ldots, Y(N)$, where $N$ is its length, we reconstruct novel time series named $Y_{k}^{m}$ calculated as

$$
Y_{k}^{m}: Y(m), Y(m+k), Y(m+2 k), \ldots, Y\left(m+\left[\frac{N-m}{k}\right] \cdot k\right),
$$

where $m$ and $k$ are two integers, $m \in[1, k]$ and [.] is the integer part.

(ii) For each $k \in\left[1, k_{\max }\right]$ and $m \in[1, k]$, we calculate the curve length as

$$
C_{m}(k)=\left(\sum_{i=1}^{[(N-m) / k]}|Y(m+i k)-Y(m+(i-1) k)|\right)\left(\frac{N-1}{[(N-m) / k] k}\right) .
$$

(iii) The length $L(k)$ is defined by

$$
L(k)=\frac{\sum_{m=1}^{k} C_{m}}{k} .
$$

(iv) In Higuchi's paper, he supposed that, for fractal time series, $L(k)$ tends towards $k^{-f}$, where $f$ is the Higuchi fractal dimension (HFD), so HFD is calculated as

$$
\mathrm{HFD}=\frac{\ln (L(k))}{\ln (1 / k)} .
$$

The main problem using HFD is the determination of $k=K_{\max }$ factor. As mentioned in equations (4)-(6), we notice that the number of program iterations depends on the chosen $K_{\max }$. In Higuchi's paper, in which he defined his algorithm, he did not give a theoretical definition, allowing to choose an exact value of the $K_{\max }$ factor. He just proposed to choose this factor through the linear regression of a log-log plot; this method is detailed in [4]. This allows to find a different value of $K_{\max }$ by studying the same signals; for example, it is chosen to be equal to 5 in $[4,7]$ and 128 in [5], for the EEG signals extracted from the same database.

\subsection{Proposed Methodology}

2.4.1. Chaos Injection. The evolution of HFD as a function of $K_{\max }$ of five EEG signals extracted from different subsets (Z, $\mathrm{O}, \mathrm{N}, \mathrm{F}$, and $\mathrm{S})$ is exposed in Figure $2(\alpha=0)$. HFD as a function of $K_{\max }$ has an increasing evolution; we notice that different curves pass through a critical point $\left(K_{\max 1} \approx 18\right)$ in which the evolution changes from important to weak. Chaos injection will be used in this work to detect signal irregularity.

We measured the HFD as a function of $K_{\max }$ for different values of $\alpha$; a selection of 3 cases $(\alpha=50, \alpha=100$, and $\alpha=150$ ) is shown in Figure 1. We notice that HFD signals outside crises ( $\mathrm{Z}, \mathrm{O}, \mathrm{N}$, and $\mathrm{F}$ ) are highly modified; nevertheless, for the $S$ signal, it is weakly modified. Moreover, we note that 


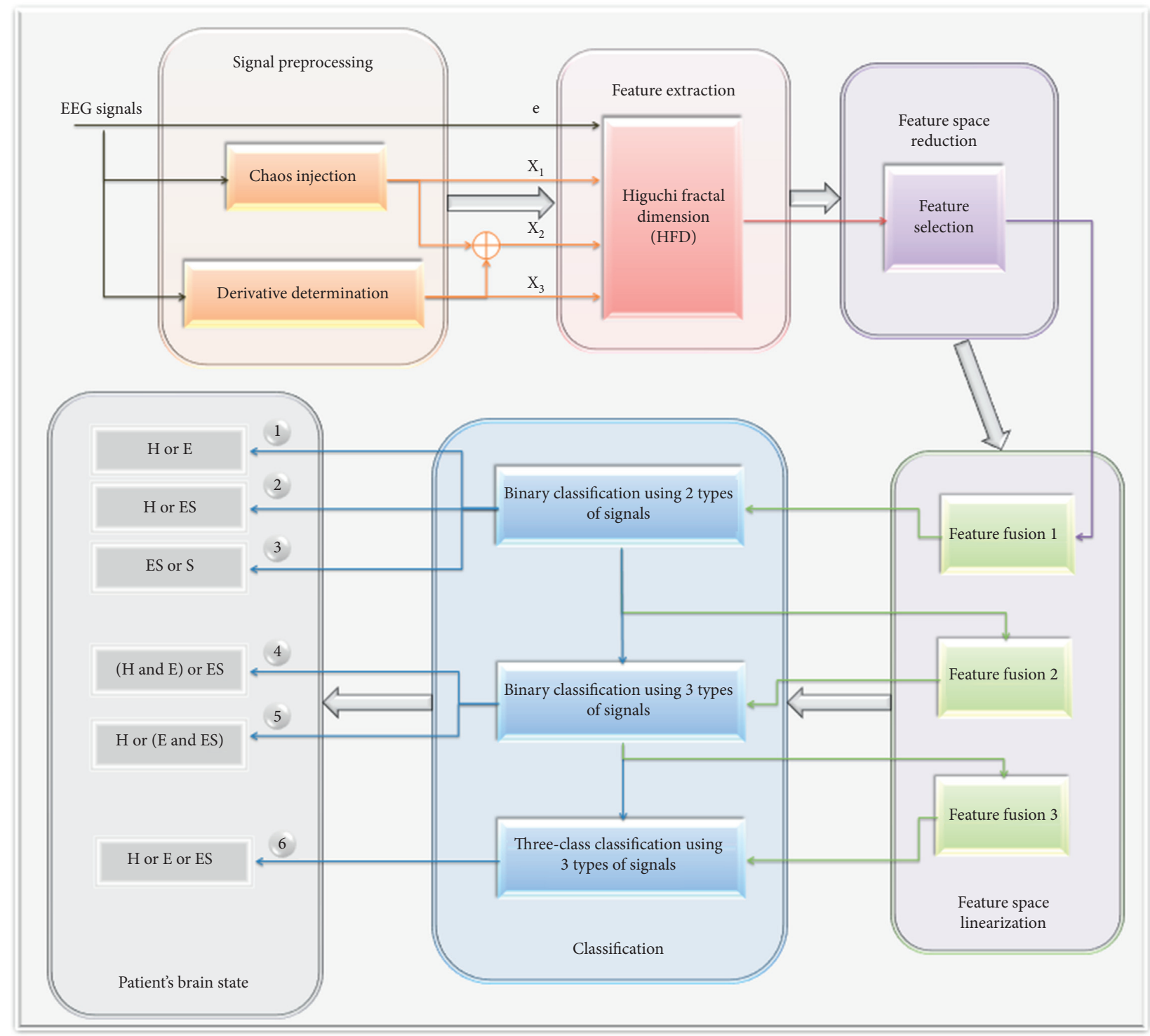

Figure 1: Illustration of the proposed machine learning (PML) model.

the curve presents a new critical point $K_{\max 2} \approx 6$, where there is a modification in the direction of change for $\mathrm{H}$ cases and the rate of change for $\mathrm{E}$ cases.

2.4.2. Derivative Exploitation. In the previous work [11], we have shown that the features extracted from the derivative of EEG have given a significant result in epilepsy diagnosis. In this paper, the HFD of the third derivative of EEG, $\operatorname{HFD}\left(x_{3}\right)$, is calculated. In Figure 3(a), we show the HFD of $e$ and $x_{1}$ (for $\alpha=100$ ), for each subset. In Figure 3(b), we show the HFD of the derivatives, $\left(x_{2}\right)$ and $\left(x_{3}\right)$, for the same sets. We note that HFD-based chaos injection applied in signal derivatives was highly modified for the ES signal.
As depicted in the previous two figures, the evolution of the fractal dimension in the function of $K_{\max }$ presents some critical points. In the follow-up of this work, we will calculate five HFDs of the signal for different conditions summarized in Table 2 .

\section{Experimental Results}

In this part, we have randomly selected $50 \%$ of signals existing in each subset for model training and the other $50 \%$ for tests.

3.1. Binary Classification Using Two Types of Signals. We used the five features introduced in Table $2\left(\mathrm{HFD}_{1}, \mathrm{HFD}_{2}, \mathrm{HFD}_{3}\right.$, $\mathrm{HFD}_{4}$, and $\mathrm{HFD}_{5}$ ). In Table 3 , we expose the results of the 

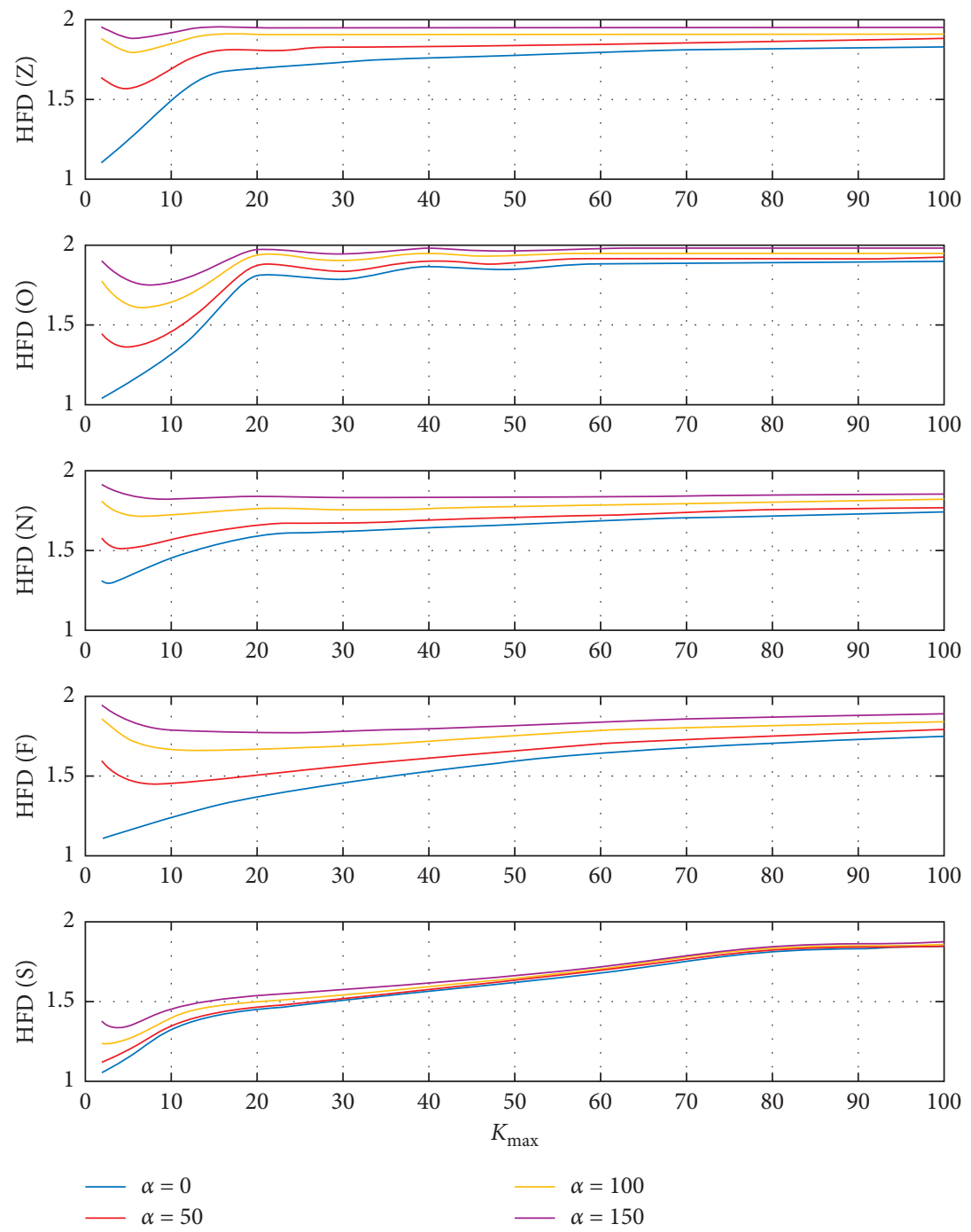

FIGURE 2: The impact of chaos injection on the variation of the Higuchi fractal dimension as a function of the $K_{\max }$ factor.

classifications of EEG signals applied to all the features, KNN (all_feat), and a subset of them, KNN (sel_feat).

(i) $\operatorname{Exp} 1$ : we have found that $\left(\mathrm{HFD}_{1}, \mathrm{HFD}_{2}\right.$, and $\left.\mathrm{HFD}_{4}\right)$ are sufficient to solve this problem. This result is illustrated in Figure 4(a), and the dispersions between the classes are clearly visible. Moreover, we have found that, for the two subproblems (ZO-N and ZO-F), the classification can be achieved by only two features.

(ii) Exp 2: $\mathrm{HDF}_{4}$ leads to a more valid classification which is shown in Figure 4(b).

(iii) Exp 3: the best classification accuracy for seizure detection is achieved using three features $\left(\mathrm{HFD}_{2}\right.$, $\mathrm{HFD}_{3}$, and $\mathrm{HFD}_{5}$ ). In Figure 4(c), we present this problem using all these features. Moreover, we find that, for the subproblems (N-S and F-S), two features are sufficient for the classifications.

\subsection{Binary and Three-Class Classification Using Three Types of Signals}

In this part, we will take advantage of the results obtained in the first three experiments. In each Exp, selected features for each case are observed in Table 4 . We are going to use feature fusion exposed in Table 4 to solve the following Exps.

(iv) Exp 4: we used $\mathrm{HFD}_{4}$ and a selection of $F F_{31}, F F_{32}$, and $F F_{3}$ according to the need for classification which is forced by subset combination requirements.

(v) Exp 5: to detect the $\mathrm{H}$ subject, for all the probabilistic set combinations, we used $\mathrm{HDF}_{4}$ and $F F_{1}$, and we have designed new Feat $F F_{5}$ as mentioned in Table 4.

(vi) Exp 6: for the three-class classification cases, we will exploit $F F$ done in $\operatorname{Exp} 4$ and $\operatorname{Exp} 5$ to represent the 

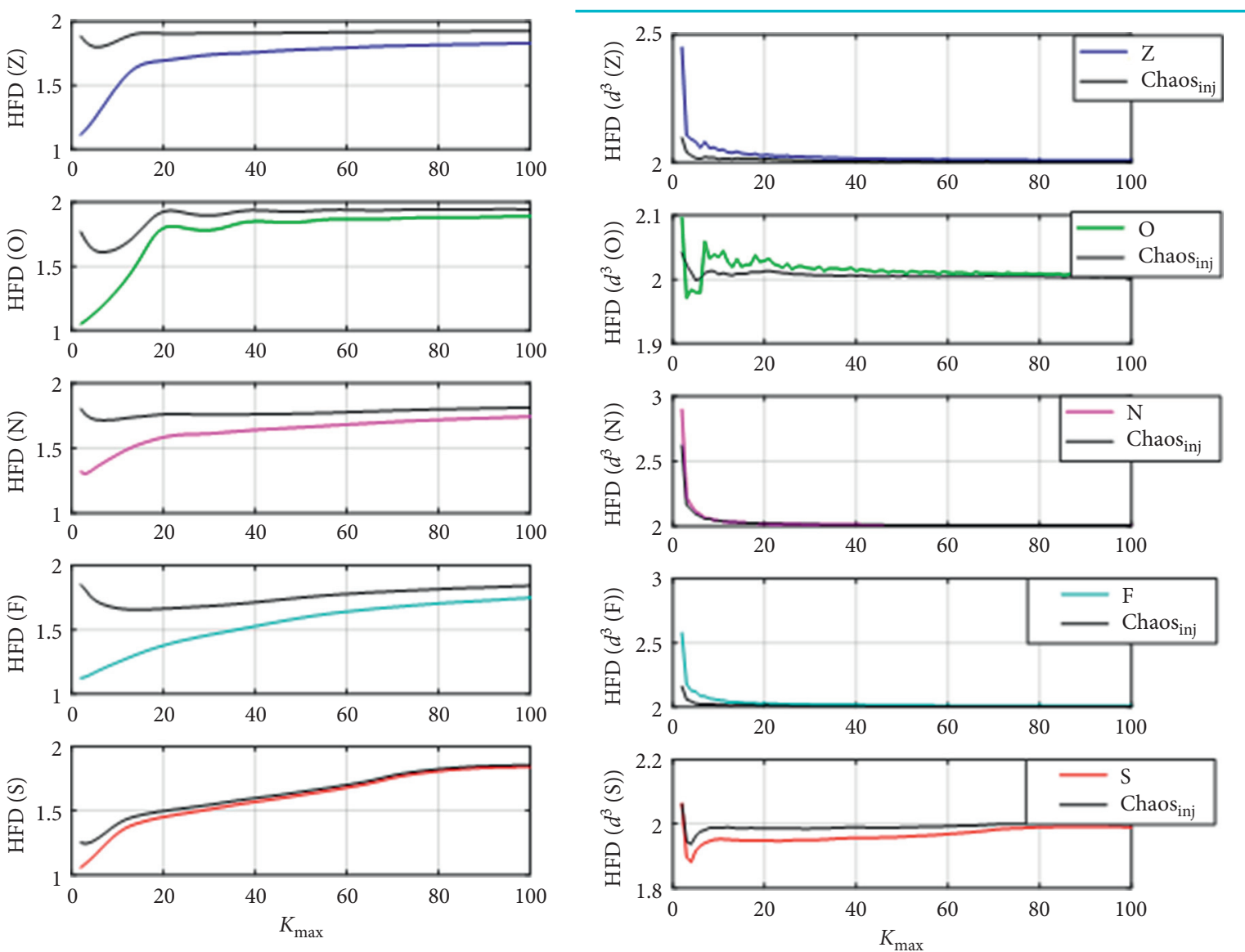

(a)

(b)

FIgure 3: Higuchi fractal dimension of the EEG signal and its third derivative.

TABLE 2: Higuchi fractal dimension of studied signals with a different selection of the $K_{\max }$ factor.

\begin{tabular}{lcc}
\hline Extracted features & Used signal & Chosen $K_{\max }$ factor \\
\hline $\mathrm{HFD}_{1}$ & $e$ & 18 \\
$\mathrm{HFD}_{2}$ & $x_{3}$ & 18 \\
$\mathrm{HFD}_{3}$ & $x_{1}$ & 6 \\
$\mathrm{HFD}_{4}$ & $x_{1}$ & 18 \\
$\mathrm{HFD}_{5}$ & $x_{2}$ & 18 \\
\hline
\end{tabular}

problem in the 2D space; FF of this Exp is shown in Table 4.

All classification problems are assessed by a $k$-nearest neighbor (KNN) classifier using all features (KNN all) and a selection of features using our proposed method (PML). Classification accuracy is exposed in Table 5. For these cases, we have also reduced the feature space which is advantageous for a real application while increasing the classification accuracy. We have shown that our method presents several advantages. Firstly, we have used a minimum number of features which vary between 1 and 5
TABLE 3: Classification accuracy (\%) using the KNN classifier applied to all features and selection of features.

\begin{tabular}{llcc}
\hline & & KNN (all_feat) & KNN (sel_feat) \\
\hline \multirow{2}{*}{ ZO-N } & Max & 100 & 100 \\
& Min & 97.33 & 98 \\
\hline \multirow{2}{*}{ ZO-F } & Max & 100 & 100 \\
& Min & 97.33 & 98 \\
\hline \multirow{2}{*}{ S-N } & Max & 100 & 100 \\
& Min & 97.33 & 98 \\
\hline \multirow{2}{*}{ S-F } & Max & 100 & 100 \\
& Min & 97.33 & 98 \\
\hline
\end{tabular}

to solve the whole classification problem using all possible combinations of subsets. In addition, the classification is made using several transformations applied to the features that reduces the dimension of the feature space; this allowed us to simplify the choice of the decision function to solve the classification problem. Moreover, the precision achieved from each Exp is of a high value. Finally, we used features calculated with a 


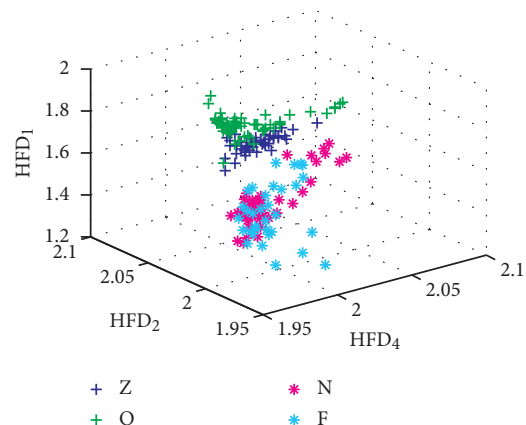

(a)

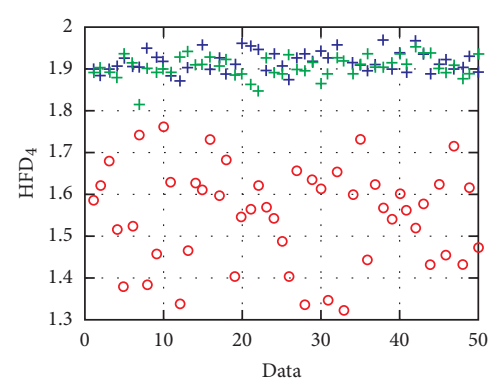

$+\mathrm{Z}$

$+\mathrm{O}$
$\circ \mathrm{S}$

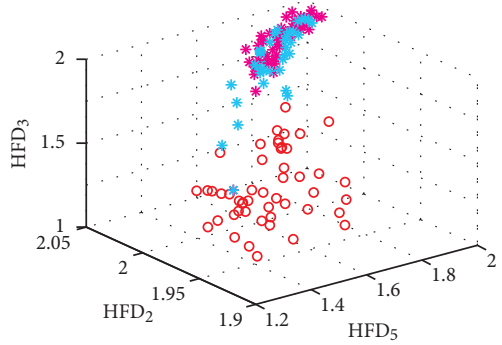

* N

* F

$\circ \mathrm{S}$

(b)

(c)

Figure 4: Presentation of different classes of studied EEG in the feature space: (a) Exp 1, (b) Exp 2, and (c) Exp 3.

TABLE 4: Selected features and proposed feature fusion for classification.

\begin{tabular}{|c|c|c|c|}
\hline Experiments & U-Sub & Input feat & $F F$ \\
\hline 1 & $\begin{array}{c}\mathrm{ZO}-\mathrm{N} \\
\mathrm{ZO}-\mathrm{F} \\
\mathrm{ZO}-\mathrm{NF}\end{array}$ & $\begin{array}{c}\mathrm{HFD}_{1}-\mathrm{HFD}_{4} \\
\mathrm{HFD}_{2}-\mathrm{HFD}_{4} \\
\mathrm{HFD}_{1}-\mathrm{HFD}_{2}-\mathrm{HFD}_{4}\end{array}$ & $\begin{array}{l}F F_{11} \\
F F_{12} \\
F F_{1} \\
\end{array}$ \\
\hline 2 & $\mathrm{ZO}-\mathrm{S}$ & $\mathrm{HFD}_{4}$ & - \\
\hline 3 & $\begin{array}{l}\text { N-S } \\
\text { F-S } \\
\text { NF-S }\end{array}$ & $\begin{array}{c}\mathrm{HFD}_{2}-\mathrm{HFD}_{5} \\
\mathrm{HFD}_{3}-\mathrm{HFD}_{5} \\
\mathrm{HFD}_{2}-\mathrm{HFD}_{3}-\mathrm{HFD}_{5}\end{array}$ & $\begin{array}{l}F F_{31} \\
F F_{32} \\
F F_{3}\end{array}$ \\
\hline 4 & $\begin{array}{c}\text { ZN-S, ON-S, ZON-S } \\
\text { ZF-S, OF-S, ZOF-S } \\
\text { ZNF-S, ONF-S, ZONF-S }\end{array}$ & $\begin{array}{l}F F_{31}-\mathrm{HFD}_{4} \\
F F_{32}-\mathrm{HFD}_{4} \\
F F_{3}-\mathrm{HFD}_{4}\end{array}$ & $\begin{array}{l}F F_{41} \\
F F_{42} \\
F F_{4}\end{array}$ \\
\hline 5 & $\begin{array}{c}\text { Z-NS Z-FS, Z-NFS, } \\
\text { O-NS, O-FE, O-NFS } \\
\text { ZO-NS, ZO-F, ZO-NFS } \\
\text { ZO-NFS }\end{array}$ & $F F_{1}-\mathrm{HFD}_{4}$ & $F F_{5}$ \\
\hline 6 & $\begin{array}{c}\text { Z-N-S,O-N-S,ZO-N-S } \\
\text { Z-F-S,O-F-S, ZO-F-S } \\
\text { Z-NF-S,O-NF-S } \\
\text { ZO-NF-S }\end{array}$ & $\begin{array}{l}F F_{41}-F F_{5} \\
F F_{42}-F F_{5} \\
F F_{4}-F F_{5}\end{array}$ & $\begin{array}{l}F F_{61} \\
F F_{62} \\
F F_{6}\end{array}$ \\
\hline
\end{tabular}

TABle 5: Comparison of classification accuracy (\%) using the KNN classifier and PML.

\begin{tabular}{|c|c|c|c|}
\hline Experiments & U-Sub & KNN (all_feat) & PML (sel_feat) \\
\hline \multirow{9}{*}{ Exp 1} & $\mathrm{Z}-\mathrm{N}$ & 96.7 & \multirow{9}{*}{100} \\
\hline & $\mathrm{Z}-\mathrm{F}$ & 99.4 & \\
\hline & Z-NF & 98 & \\
\hline & $\mathrm{O}-\mathrm{N}$ & 99.1 & \\
\hline & $\mathrm{O}-\mathrm{F}$ & 99.5 & \\
\hline & $\mathrm{O}-\mathrm{NF}$ & 99.8 & \\
\hline & $\mathrm{ZO}-\mathrm{N}$ & 99.13 & \\
\hline & $\mathrm{ZO}-\mathrm{F}$ & 98.46 & \\
\hline & ZO-NF & 98.6 & \\
\hline \multirow{3}{*}{ Exp 2} & Z-S & 100 & \multirow{3}{*}{100} \\
\hline & $\mathrm{O}-\mathrm{S}$ & 99.9 & \\
\hline & $\mathrm{ZO}-\mathrm{S}$ & 100 & \\
\hline \multirow{3}{*}{ Exp 3} & $\mathrm{~N}-\mathrm{S}$ & 98.6 & 100 \\
\hline & F-S & 97.1 & 99 \\
\hline & NF-S & 97.73 & 99.5 \\
\hline
\end{tabular}


TABLE 5: Continued.

\begin{tabular}{|c|c|c|c|}
\hline Experiments & U-Sub & KNN (all_feat) & PML (sel_feat) \\
\hline \multirow{9}{*}{ Exp 4} & ZN-S & 99.26 & 100 \\
\hline & ZF-S & 98.2 & 98 \\
\hline & ZNF-S & 98.25 & 99 \\
\hline & ON-S & 99.33 & 100 \\
\hline & OF-S & 98 & 98 \\
\hline & ONF-S & 98.05 & 99.4 \\
\hline & ZON-S & 99.5 & 100 \\
\hline & ZOF-S & 98.65 & 99.4 \\
\hline & ZONF-S & 98.76 & 99.6 \\
\hline \multirow{9}{*}{ Exp 5} & Z-NS & 99.8 & \multirow{9}{*}{100} \\
\hline & Z-FS & 98.9 & \\
\hline & Z-NFS & 98.5 & \\
\hline & O-NS & 99.46 & \\
\hline & O-FS & 99 & \\
\hline & O-NFS & 98.8 & \\
\hline & ZO-NS & 99.65 & \\
\hline & ZO-FS & 99.05 & \\
\hline & ZO-NFS & 98.96 & \\
\hline \multirow{9}{*}{ Exp 6} & Z-N-S & 99.26 & 100 \\
\hline & Z-F-S & 96.2 & 99.2 \\
\hline & Z-NF-S & 96.65 & 99.6 \\
\hline & $\mathrm{O}-\mathrm{N}-\mathrm{S}$ & 98.4 & 100 \\
\hline & O-F-S & 97.4 & 99.25 \\
\hline & O-NF-S & 97.3 & 99.62 \\
\hline & ZO-N-S & 99.25 & 100 \\
\hline & ZO-F-S & 97.5 & 99.42 \\
\hline & ZO-NF-S & 97.28 & 99.54 \\
\hline
\end{tabular}

TABLe 6: Duration in seconds for the extraction of each feature.

\begin{tabular}{lc}
\hline Extracted feature & Elapsed time in seconds \\
\hline $\mathrm{HFD}_{1}$ & 0.02 \\
$\mathrm{HFD}_{2}$ & 0.02 \\
$\mathrm{HFD}_{3}$ & 0.01 \\
$\mathrm{HFD}_{4}$ & 0.02 \\
$\mathrm{HFD}$ & \\
\hline
\end{tabular}

simple algorithm whose calculation time of each one is shown in Table 6. Experimental tests were performed using Intel ${ }^{\circledR}$ Xeon Dual-Core W3550 $3.06 \mathrm{GHz}$.

\section{Discussion}

At this level, it is important to evaluate the performance of our method compared to other published works that use the same database. In Table 7, we present the accuracy of various selected works of the state of the art by indicating the machine learning methods, the number of used features $\left(N_{\text {feat }}\right)$,the combinations of subsets used (U-Sub), and the publication date (PY) of each reference (Ref).

According to these state-of-the-art papers, two main methodologies were used to achieve high accuracy in all experiments as mentioned in Table 7. The first one is fractal theory and nonlinear analysis for feature extraction, which are considered efficient tools to study EEG signals in different cited papers; they used various signal preprocessing methods. Scattering transform in [10] was used to extract 14 nonlinear features. A nonlinear analysis based on multifractal decomposition in [9] was used to extract 14 features. HFD was used to extract 17 features in [5] and 13 features in [6] from, respectively, two different wavelet decompositions ATFFWT and TQFWT. In addition, more than HFD, other nonlinear features were extracted from EEG using different other signal decomposition methods, FFT in [4] to extract 38 features, LMD in [7] to extract 9 features, and BOWT in [8] to extract 25 features. The second one is the signal derivative in the preprocessing step, which is presented in our previous work; we showed that derivatives contain important information about EEG signals. In fact, using derivatives in the signal preprocessing step, we used few features in all classifications experiments, but those results are valid only for a limited combination of used subsets. In this paper, HFD and derivative determination were applied to design an efficient machine learning model that overcomes all the accuracies achieved in cited works. Although we use a minimal number of features compared to other works, for each Exp, only in this work, an accuracy of $100 \%$ was reached for some or all of the possible combinations of subsets of each case. 
TABLE 7: Comparison of achieved accuracy by our method and other published methods using the Bonn database.

\begin{tabular}{|c|c|c|c|c|c|}
\hline Exps & Ref-PY & Methods & $N_{\text {feat }}$ & U-Sub & Acc $(\%)$ \\
\hline \multirow{7}{*}{1} & {$[4], 2008$} & FFT-RIR-PFD-HFD-HPs-PNN & 38 & ZO-NF & 99.5 \\
\hline & [5], 2017 & ATFFWT-HFD-SVM & 17 & $\mathrm{ZO}-\mathrm{NF}$ & 92.5 \\
\hline & {$[6], 2017$} & TQFWT-HFD-SVM & 13 & $\mathrm{ZO}-\mathrm{NF}$ & 98.5 \\
\hline & [9], 2018 & MFA-SVM & 14 & $\mathrm{ZO}-\mathrm{NF}$ & 96.25 \\
\hline & [7], 2019 & BOWT-HFD-NLfeats-En-SVM & 25 & $\mathrm{ZO}-\mathrm{NF}$ & 99 \\
\hline & {$[11], 2020$} & Derivative-LogVar-Kt & 2 & $\mathrm{Z}-\mathrm{N}$ & 100 \\
\hline & This work & HFD-PML & $\begin{array}{l}2 \\
3\end{array}$ & $\begin{array}{c}\mathrm{Z}-\mathrm{N} / \mathrm{O}-\mathrm{N} / \mathrm{ZO}-\mathrm{N} / \mathrm{Z}-\mathrm{F} / \mathrm{O}-\mathrm{F} / \mathrm{ZO}-\mathrm{F} \\
\mathrm{Z}-\mathrm{NF} / \mathrm{O}-\mathrm{NF} / \mathrm{ZO}-\mathrm{NF}\end{array}$ & 100 \\
\hline \multirow{9}{*}{2} & {$[4], 2008$} & FFT-RIR-PFD-HFD-HPs-PNN & 38 & $\mathrm{ZO}-\mathrm{S}$ & 98.3 \\
\hline & [5], 2017 & ATFFWT-HFD-SVM & $7 / 8 / 16$ & $\mathrm{Z}-\mathrm{S} / \mathrm{O}-\mathrm{S} / \mathrm{ZO}-\mathrm{S}$ & 100 \\
\hline & [6], 2017 & TQFWT-HFD-SVM & $10 / 5 / 8$ & Z-S/O-S/ZO-S & 100 \\
\hline & [7], 2019 & BOWT-HFD-NLfeats-En-SVM & 25 & $\mathrm{ZO}-\mathrm{S}$ & 99 \\
\hline & {$[8], 2016$} & LMD-HFD-HE-ReEn-TSFs-SVM & 9 & Z-S & 100 \\
\hline & [9], 2018 & MFA-SVM & 14 & $\mathrm{ZO}-\mathrm{S}$ & 100 \\
\hline & {$[10], 2020$} & ScT-LogEF-FuzEn-SVM & 14 & Z-S/O-S/ZO-S & 100 \\
\hline & {$[11], 2020$} & Derivative-LogVar-Kt & 2 & Z-S & 99.8 \\
\hline & This work & HFD-PML & 1 & $\mathrm{Z}-\mathrm{S} / \mathrm{O}-\mathrm{S} / \mathrm{ZO}-\mathrm{S}$ & 100 \\
\hline \multirow{9}{*}{3} & [4], 2008 & FFT-RIR-PFD-HFD-HPs-PNN & 38 & NF-S & 96.7 \\
\hline & [5], 2017 & ATFFWT-HFD-SVM & 17 & N-S/F-S/NFS & 92.5 \\
\hline & {$[6], 2017$} & TQFWT-HFD-SVM & $7 / 9 / 10$ & N-S/F-S/NFS & 98.5 \\
\hline & [7], 2019 & BOWT-HFD-NLfeats-En-SVM & 25 & NF-S & 99 \\
\hline & [8], 2016 & LMD-HFD-HE-ReEn-TSFs-SVM & 9 & F-S & 98.10 \\
\hline & [9], 2018 & MFA-SVM & 14 & NF-S & 98.3 \\
\hline & {$[10], 2020$} & ScT-LogEF-FuzEn-SVM & 14 & N-S/(F-S, NF-S) & $100 / 99.96$ \\
\hline & {$[11], 2020$} & Derivative-LogVar-Kt & 2 & $\mathrm{~N}-\mathrm{S}$ & 100 \\
\hline & This work & HFD-PML & 2 & $\mathrm{~N}-\mathrm{S}$ & 100 \\
\hline \multirow{8}{*}{4} & {$[8], 2016$} & LMD-HFD-HE-ReEn-TSFs-SVM & 9 & ZONF-S & 100 \\
\hline & [5], 2017 & ATFFWT-HFD-SVM & 17 & ZONF-S & 92.2 \\
\hline & {$[6], 2017$} & TQFWT-HFD-SVM & 10 & ZONF-S & 99.6 \\
\hline & [7], 2019 & BOWT-HFD-NLfeats-En-SVM & 25 & ZO-NF & 99 \\
\hline & [9], 2018 & MFA-SVM & 14 & ZONF-S & 98.25 \\
\hline & {$[10], 2020$} & ScT-LogEF-FuzEn-SVM & 14 & ZONF-S & 100 \\
\hline & {$[11], 2020$} & Derivative-LogVar-Kt & 2 & ZN-S & 100 \\
\hline & This work & HFD-PML & 3 & ZN-S/ON-S/ZON-S & 100 \\
\hline \multirow{6}{*}{5} & [7], 2019 & BOWT-HFD-NLfeats-En-SVM & 25 & ZO-NF & 99 \\
\hline & {$[10], 2020$} & ScT-LogEF-FuzEn-SVM & 14 & ZO-NFS & 100 \\
\hline & {$[11], 2020$} & Derivative-LogVar-Kt & 2 & Z-N-S & 99.85 \\
\hline & & & & Z-NS/O-NS/ZO-NS & \\
\hline & This work & HFD-PML & 3 & Z-FS/O-FS/ZO-FS & 100 \\
\hline & & & 4 & Z-NFS/O-NFS/ZO-NFS & \\
\hline \multirow{5}{*}{6} & {$[8], 2016$} & LMD-HFD-HE-ReEn-TSFs-SVM & 9 & Z-F-S/ZO-NF-S & $98.47 / 98.4$ \\
\hline & [7], 2019 & BOWT-HFD-NLfeats-En-SVM & 25 & ZO-NF-S & 98.4 \\
\hline & {$[10], 2020$} & ScT-LogEF-FuzEn-SVM & 14 & Z-F-S/ZO-NF-S & $99.10 / 99.87$ \\
\hline & {$[11], 2020$} & Derivative-LogVar-Kt & 2 & Z-N-S & 98.5 \\
\hline & This work & HFD-PML & $4 / 5$ & Z-N-S/O-N-S/ZO-N-S & 100 \\
\hline
\end{tabular}

\section{Conclusion}

An innovative EEG signal processing method for the automatic detection of epilepsy and epileptic seizures was presented in this paper. We suggest a new method for signal preprocessing using EEG derivative determination and chaotic signal injection. In addition, we propose a new method for Higuchi fractal dimension determination. The experimental results proved the efficiency of our strategy, and we achieved a significant accuracy with few features and with minimum execution time compared to other published works. Our future direction will concern about how to profit from the richness of the two theories, fractal and chaos, for analyzing EMG and ECG to propose an accurate epilepsy diagnostic system based on the multisensor system.

\section{Data Availability}

No data were used to support this study. 


\section{Conflicts of Interest}

The authors declare that there are no conflicts of interest regarding the publication of this paper.

\section{References}

[1] A. L. Goldberger, D. R. Rigney, and B. J. West, "Science in pictures: chaos and fractals in human physiology," Scientific American, vol. 262, no. 2, pp. 42-49, 1990.

[2] C. A. Skarda and W. J. Freeman, "How brains make chaos in order to make sense of the world," Behavioral and Brain Sciences, vol. 10, no. 2, pp. 161-173, 1987.

[3] J. P. Pijn, J. Van Neerven, A. Noest, and F. H. Lopes da Silva, "Chaos or noise in EEG signals; dependence on state and brain site," Electroencephalography and Clinical Neurophysiology, vol. 79, no. 5, pp. 371-381, 1991.

[4] F. S. Bao, D. Y.-C. Lie, and Y. Zhang, "A new approach to automated epileptic diagnosis using EEG and probabilistic neural network," in Proceedings of the 2008 20th IEEE International Conference on Tools with Artificial Intelligence, IEEE, Dayton, OH, USA, November 2008.

[5] M. Sharma, R. B. Pachori, and U. Rajendra Acharya, "A new approach to characterize epileptic seizures using analytic time-frequency flexible wavelet transform and fractal dimension," Pattern Recognition Letters, vol. 94, pp. 172-179, 2017.

[6] M. Sharma and R. B. Pachori, "A novel approach to detect epileptic seizures using a combination of tunable-q wavelet transform and fractal dimension," Journal of Mechanics in Medicine and Biology, vol. 17, no. 7, Article ID 1740003, 2017.

[7] M. Sharma, S. Shah, and P. V. Achuth, "A novel approach for epilepsy detection using time-frequency localized Bi-orthogonal wavelet filter," Journal of Mechanics in Medicine and Biology, vol. 19, no. 1, Article ID 1940007, 2019.

[8] T. Zhang and W. Chen, "LMD based features for the automatic seizure detection of EEG signals using SVM," IEEE Transactions on Neural Systems and Rehabilitation Engineering, vol. 25, no. 8, pp. 1100-1108, 2016.

[9] R. Bose, S. Pratiher, and S. Chatterjee, "Detection of epileptic seizure employing a novel Set of features extracted from multifractal Spectrum of electroencephalogram signals," IET Signal Processing, vol. 13, no. 2, pp. 157-164, 2018.

[10] Y. Jiang, W. Chen, and Y. You, "Scattering transform-based features for the automatic seizure detection," Biocybernetics and Biomedical Engineering, vol. 40, no. 1, pp. 77-89, 2020.

[11] Z. Brari and S. Belghith, "A new method for the detection of epilepsy andepileptic seizures based on the variance of EEG signals and its derivatives with a simple kernel trick," in Proceedings of the 2020 4th International Conference on Advanced Systems and Emergent Technologies (IC_ASET), Hammamet, Tunisia, December 2020.

[12] R. G. Andrzejak, K. Lehnertz, F. Mormann, C. Rieke, P. David, and C. E. Elger, "Indications of nonlinear deterministic and finite-dimensional structures in time series of brain electrical activity: dependence on recording region and brain state," Physical Review E, vol. 64, no. 6 Pt 1, Article ID 061907, 2001.

[13] M. J. Woyshville and J. R. Calabrese, "Quantification of occipital EEG changes in Alzheimer's disease utilizing a new metric: the fractal dimension," Biological Psychiatry, vol. 35, no. 6, pp. 381-387, 1994.

[14] T. Higuchi, "Approach to an irregular time series on the basis of the fractal theory," Physica D: Nonlinear Phenomena, vol. 31, no. 2, pp. 277-283, 1988.
[15] S. Kesić and S. Z. Spasić, "Application of Higuchi's Fractal Dimension from basic to clinical neurophysiology: a review," Computer Methods and Programs in Biomedicine, vol. 133, pp. 55-70, 2016. 